\title{
AEROBIC PYOGENIC LIVER ABSCESS
}

\author{
Col HARIQBAL SINGH *, Maj MANDEEP SAINI ${ }^{+}$, \\ Maj VINAY MAURYA ${ }^{\#}$, Lt Col L SATIJA ${ }^{* *}$
}

MJAFI $2001 ; 57: 80-81$

KEY WORD :Liver abscess.

\section{Introduction}

$\mathbf{L}$ iver abscesses of bacterial origin are less frequent than parasitic and those due to gas producing organisms are uncommon. Pyogenic liver abscess can have an acute presentation with a rapid fulminant course. We present a case with subtle radiological features developing within 48 hours into a full-blown classical pyogenic liver abscess due to gas producing organism. Despite continued advances in diagnostic imaging and treatment, hepatic abscess remains a serious problem. CT scan is a sensitive and most accurate imaging modality for detecting hepatic abscess but is often non specific [1].

\section{Case Report}

50 year old postmenopansal female presented with complaints of fever with chills and rigors and generalized abdominal distension of 10 days duration, vague pain abdomen, occasional vomiting and constipation of two days duration. On examination she was found to be febrile (temp $99.6^{\circ} \mathrm{F}$ ) with a BP of $110 / 70 \mathrm{~mm}$ of Hg. She had no jaundice but had mild pallor with non-tender hepatomegaly of $8 \mathrm{~cm}$ below the right subcostal margin and bilateral basal crepitations.

On investigation she had $\mathrm{Hb}-10.5 \mathrm{gm} \%$ with polymorphonu-

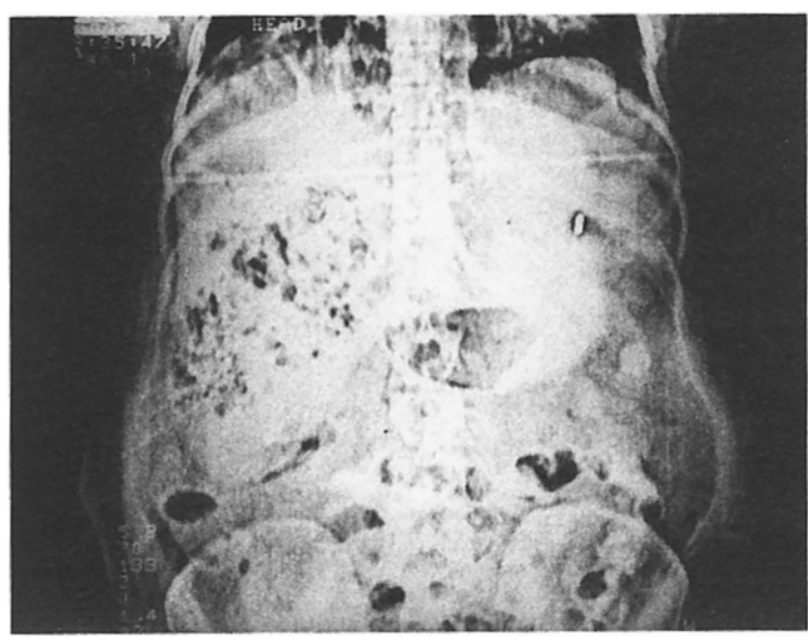

Fig. 1: Topogram reveals multiple air pockets in right lobe of liver. clear leucocytosis, serum bilirubin - $3.2 \mathrm{gm} \%$ (direct positive) with mildly raised liver enzymes, blood urea- $60 \mathrm{mg} \%$ and serum creatinine $-2.6 \mathrm{mg} \%$ with a random blood sugar of $360 \mathrm{mg} \%$.

Ultrasound revealed hepatomegaly with a large il]-defined area of altered echogenicity in the right lobe, there was no djlation of intra hepatic biliary radicles. Multiple calculi were seen in the galibladder with no evidence of ascites or retroperitoneal lymphadenopathy, a large uterine fibroid with hydrometra was also demonstrated. Ultrasound repeated after 24 hours revealed a large jso to hypoechoic lesion with multiple tiny bright echoes with a characteristic bright comet tail distal artefact in the right lobe. The patient was being managed as a case of septicaemia with liver abscess. With continued deterioration in patient's condition on subsequtent day a CT scan was contemplated, the topogram revealed enlarged liver with multiple pockets of air in the right lobe (Fig 1). The axial sections revealed multiple gas containing pockets several of which were trident shaped within the abscess area seen as relatively low attenuating area having CT value of 38-47 $\mathrm{HU}$ as against the normal liver parenchyma having $\mathrm{CT}$ value as $70-78 \mathrm{HU}$, measuring $11 \times 9 \times 10 \mathrm{~cm}$ in the right lobe of liver. There was a breach in the liver parenchyma limited by hepatic capsule along the lateral surface (Fig 2). A diagnosis of pyogenic liver abscess due to gas producing organism with impending rupture was offered. Ultrasound guided aspiration yielded about 100 $\mathrm{ml}$ of thick reddish-brown purulent material with gas bubbles.

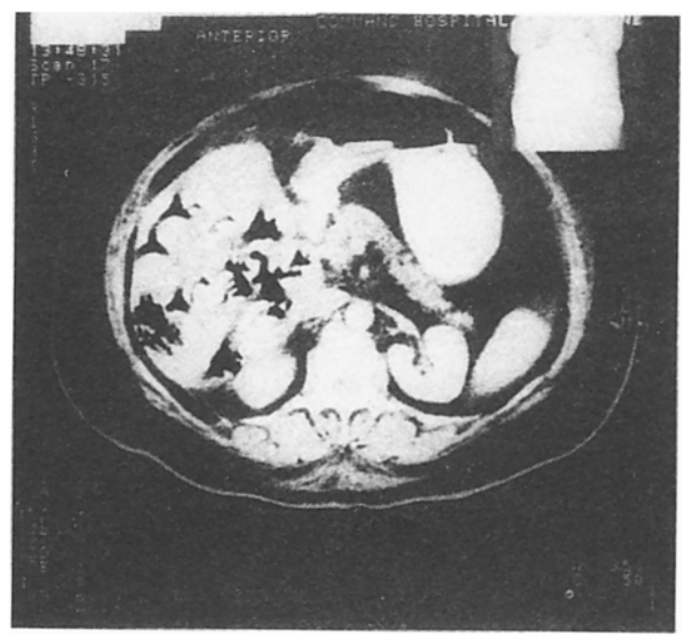

Fig. 2: CT scan abdomen, sections through right lobe of liver show multiple pockets of air in the abscess area with breach in liver parenchyma limited by hepatic capsule along the lateral surface

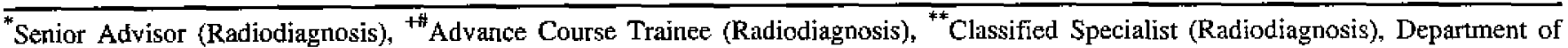
Radiodiagnosis, Command Hospital (Southern Command), Pune - 411040.
} 
On laparotomy liver was grossly enlarged, tense and congested with multiple recrotic areas having purulent collection. Gall bladder was packed with mulberty stones and pus. CBD appeared dilated with thickened wall. However there was no evidence of peritonitis or pancreatitis. Deroofing of the abscess cavity with open drainage with cholecystectomy and thorough peritoneal layage was performed.

Culture of the liver aspirate showed growth of Klebsiella aerogenes, which is a gas producing organism [2]. Patient responded to appropriate antibiotics and posioperative period was uneventful.

\section{Discussion}

The liver is the organ most stibject to development of abscesses, of all visceral abscesses $48 \%$ are liver abscess [3]. In developing countries liver abscesses are mmmon, most represent parasitic infections eg., amocbic, echinococcal or other protozoal or helminthic organisms. In developed countries these are becoming uncommon. Most liver abscess of bacterial origin represent a secondary complication of an infection elsewhere. The organisms reach the liver through one of the following pathways : (a) biliary, due to ascending cholangitis; (b) portal, due to bactermia occurring in intra abdominal sepsis; (c) arterial, due to septicemia; (d) local due to suppuration involving neighbouring tissues; and (e) penetrating injuries [4].

Depending on their pathogenesis, pyogenic hepatic abscesses may occur as solitary or multiple lesions ranging in size from few millimeters to massive lesions as seen in this case. Liver abscesses, whatever their origin, are associated with fever and in many instances right upper quadrant pain, tender hepatomegaly and uncommonly jaundice [3].

Imaging studies are the most reliable method for diagnosing liver abscess. These include ultrasound, CT scan, gallium scan and even magnetic resonance imaging. In an occasional case, more than one such study may be required. Sonography has proved to be extremely helpful in detection of liver abscesses. The ultrasound features of pyogenic liver abscesses are varied. Frankly purulent abscesses appear cystic, with the fluid ranging from echo-free to highly echogenic.
Regions of early suppuration may appear solid with altered echogenicity, usually hypoechoic related to the presence of necrotic hepatocytes [5]. Gas producing organisms give rise to bright echos with comet tail artefact [6]. Fluid-fluid interfaces, internal septations and debris have all been observed. The abscess wall can vary from well defined to irregular or thick walled. Differential diagnosis of pyogenic liver abscess includes amoebic or echinococcal infection, simple cyst with haemorrhage, haematoma and necrotic or cystic neoplasm. Ultrasound guided liver aspiration is an expeditious means to confirm the diagnosis. The specimen should be sent for both aerobic and anaerobic culture [7]. CT scan is the most accurate method of detection available, its excellent depiction of anatomic detail allows for precise localization of abnormal fluid collection [1]. Unfortunately, however, the CT appearances can be nonspecific and aspiration biopsy with microbiological confirmation is usually necessary for diagnosis. When gas is present with an abnormal fluid collection or a low attenuation area is seen within the liver, the diagnosis of abscess can be made with high degree of confjdence.

\section{References}

1. Halversen RA, Korobkin M, Foster WL, The variable CT appearance of hepatic abscesses. AJR 1984;141:94I-3.

2. Cowan and Steel's Manual for identification of medical bacteria. 3rd ed Cambridge University Press. 1993;3:138-9.

3. Dori F, Zaleznick, Dennis LK. Intra-abdominal infections and abscesses in Harrison's principles of internal medicine, McGraw Hill,14th ed. 1998;Ch127.

4. Mathieu D, Vasile N, Fagneiz PL, Segui S, Grably D, Larde D. Dynamic CT features of hepatic abscess. Radiology 1985 ; 154:749-52.

5. Wilson SR, Arenson AM. Sonographic evaluation of hepatic abscesses. J Can Assoc Radiol 1984;35:174-7.

6. Ralls PW, Bames PF, Quinn MF. Sonographic features of amoebic and pyogenic liver abscesses : a blinded comparison. AJR 1987;149:499-501.

7. Cynthia EW, Wilson SR. The liver:Rumack's Diagnostic ultrasound, Mosby, 1988;2:103 4 . 